\title{
Spin-glass charge ordering in ionic liquids
}

\author{
Amir Levy, ${ }^{1}$ Michael McEldrew, ${ }^{2}$ and Martin Z. Bazant ${ }^{2,3}$ \\ ${ }^{1}$ Department of Physics, Massachusetts Institute of Technology, Cambridge, Massachusetts 02139, USA \\ ${ }^{2}$ Department of Chemical Engineering, Massachusetts Institute of Technology, Cambridge, Massachusetts 02139, USA \\ ${ }^{3}$ Department of Mathematics, Massachusetts Institute of Technology, Cambridge, Massachusetts 02139, USA
}

(Received 20 August 2018; revised manuscript received 6 March 2019; published 20 May 2019)

\begin{abstract}
Ionic liquids form intricate nanostructures, both in the bulk and near charged surfaces. We show that given the ionic positions from molecular simulations, the ionic charges minimize a "spin-glass" Hamiltonian for nearest-neighbor interactions with remarkable accuracy, for both room-temperature ionic liquids and water-insalt electrolytes. Thus, long-range charge oscillations in ionic liquids result from positional ordering, which is maximized in ionic solids but gradually disappears with added solvent, increased temperature, or by complex molecular structures. As the electrolyte becomes more disordered, geometrical frustration in the spin-glass ground state reduces correlation lengths. Eventually, thermal fluctuations excite the system from its ground state and Poisson-Boltzmann behavior is recovered. More generally, spin-glass ordering arises in any liquid with antiferromagnetic correlations, such as molten salt or the two-dimensional vortex patterns found in superfluids and bacterial turbulence.
\end{abstract}

DOI: 10.1103/PhysRevMaterials.3.055606

\section{INTRODUCTION}

In recent years, room-temperature ionic liquids (RTILs) have emerged as promising electrolytes for synthetic chemistry and electrochemical energy storage [1-5]. In the absence of solvent molecules, strong electrostatic interactions limit the applicability of classical mean-field approximations, such as the ubiquitous Poisson-Boltzmann (PB) theory [6] for dilute solutions. Extensions are available for steric effects [7-11], short-range ion-ion forces [12-17], ion-solvent interactions $[18,19]$, and Gaussian perturbations beyond mean field [20], but no theory can fully describe the solvent-free limit of RTILs.

At electrified interfaces, ionic liquids share similarities with dilute electrolytes, and some aspects can be described by modified continuum models. Direct surface force measurements reveal a diffuse electric double-layer (EDL) structure, akin to that of a dilute aqueous solution [21], although the extent of this analogy is debated [22]. Nevertheless, there have been some successful applications of mean-field continuum models to RTILs $[1,11,23]$. Additionally, strong electrostatic correlations, which induce charge ordering and oscillations [24], can be captured surprisingly well by higher-order PBtype equations [25-31].

Strong charge correlations imply a nonlocal dielectric response, similar to that of polar solvents [32,33]. Bazant, Storey, and Kornyshev (BSK) extended the PB free-energy functional to include both correlations and crowding effects and introduced the concept of a dielectric permittivity operator to approximate the nonlocal ionic polarization [27]. The BSK framework was subsequently used to describe a wide variety of structural [34-37] and dynamical [38-42] properties of ionic liquids and concentrated electrolytes. Continuum models continue to extend their predictive power to capture aspects of both long-range underscreening and overscreening $[15,17,43-45]$, yet they remain incomplete in their description of complex many-body correlation effects. Other phenomena, such as charge-driven three-dimensional (3D) structures of the double layer [46-49], are not well explained with a meanfield approach, and coarse-grained charge profiles generally obscure correlated nanostructures [50-52].

In this paper, we use molecular dynamics (MD) simulations to reveal an essential and overlooked mechanism that determines the charge profile in ionic liquids: geometrical frustration. Given the network of neighboring ionic positions in a symmetric binary mixture, we show that the charge distribution corresponds to the ground state of an effective spin-glass Hamiltonian [53]. We propose a minimization scheme based on a modified Goemans-Williamson (GW) algorithm [54] and perform spin-glass reconstructions of MD simulations of four materials: 1-Ethyl-3-methylimidazolium bis(trifluoromethylsulfonyl)imide (EMIM-TFSI), a commonly studied aprotic RTIL [55-58], protic RTIL trimethylammonium-triflate (TMA-OTF), molten sodium chloride, and so-called water-in-salt electrolytes (WiSE), recently introduced for Li-ion batteries [59-61]. Finally, we consider turbulence in bacterial suspensions [62] and illustrate how spin-glass ordering emerges in any strongly interacting antiferromagnetic disordered system.

\section{THEORY}

The partition function of ionic liquids (neglecting nonidealities) can be written as a sum over all spatial configurations $\left(\left\{\mathbf{r}_{i}\right\}\right)$ and valencies $\left(z_{i}\right)$ :

$$
\begin{aligned}
Z & =\int \prod_{i=1}^{N} d \mathbf{r}_{i}\left[\sum_{\left\{z_{i}\right\}} \exp \left(-l_{B} \sum_{i \neq j} \frac{z_{i} z_{j}}{\left|\mathbf{r}_{i}-\mathbf{r}_{j}\right|}\right)\right] \\
& =\int \prod d \mathbf{r}_{i} Z_{r}\left[\left\{\mathbf{r}_{i}\right\}\right],
\end{aligned}
$$


where $l_{B}=\beta e^{2} / \varepsilon$ is the Bjerrum length, $e$ is the elementary charge, $\beta=\left(k_{B} T\right)^{-1}$ is the inverse temperature, and $\varepsilon$ is the dielectric constant of the medium. $Z_{r}$ is a reduced partition function that depends on the ionic positions. We emphasize that Eq. (1) is purposely simplified, excluding any nonelectrostatic physics, such as asymmetric molecular shapes, sizes, and/or interactions, which are vital in inducing positional disorder in the fluid $[63,64]$. We may exclude such effects because once the positions are given, as in this framework, nonelectrostatic physics play only a minor role in charge ordering.

The reduced partition function is similar to a spin glass, with the following Hamiltonian:

$$
H=\frac{1}{2} \sum_{i \neq j} J_{i j} z_{i} z_{j}, \quad \text { where } \quad J_{i j}=\frac{l_{B}}{\left|\mathbf{r}_{i}-\mathbf{r}_{j}\right|} .
$$

In the dilute limit $\left(l_{B} \rightarrow 0\right)$, the Debye-Huckel mean-field approximation becomes valid, at rather small salt concentrations $(<100 \mathrm{mM})$ for aqueous solutions $\left(l_{B} \approx 7 \AA\right.$ ). In the opposite limit $\left(l_{B} \rightarrow \infty\right)$ relevant for ionic liquids, when the Bjerrum length is large compared to the ionic spacing, temperatureinduced charge fluctuations around the ground state are negligible, and the charge distribution is better approximated by minimizing the Coulomb energy.

Minimizing a spin-glass Hamiltonian is a well-known NPcomplete problem [65] that cannot be solved exactly. The difficulty lies in the "Ising-like" constraint on the charges: $z_{i}= \pm 1$, which can be expressed efficiently via a matrix, $Z_{i j}=z_{i} z_{j}$. By construction, the rank of $Z$ equals 1 and its diagonal is $Z_{i i}=1$. The Hamiltonian, in terms of $Z$, is simply $\operatorname{Tr}(Z J)$. Relaxing the constraint on the rank of $Z$, and letting it take a full rank, greatly simplifies the problem and allows for a polynomial time solution. This is the celebrated GoemansWilliamson (GW) max-cut algorithm [54]. In the context of spin glasses, the GW algorithm can be interpreted as letting the spins rotate in an $N$-dimensional space, where $N$ is the total number of spins in the system [66].

The GW algorithm steps are as follows: (1) Minimize $\operatorname{Tr}(Z J)$ subject to $Z_{i i}=1$; (2) find the Cholesky decomposition of $Z\left(Z=S S^{T}\right)$; and (3) choose a plane in the $N$ dimensional space and assign the $i$ th Ising spin a sign (charge) according to the side of the plane where the $N$-dimensional spin $S_{i k}$ lies. To solve the minimization problem, we use CVX, a package for specifying and solving convex programs $[67,68]$.

The GW algorithm can be applied to any pairwise interaction and, interestingly, we find that fully connected systems yield poor results. Instead, a dramatic improvement is achieved by considering an effective Hamiltonian with only short-range interactions, such as the following (empirical) interaction between an ion and its $n^{\text {th }}$-nearest neighbor:

$$
J_{n}^{\text {eff }}= \begin{cases}e^{-n}, & n=1 \ldots 5 \\ 0, & n>5 .\end{cases}
$$

Due to screening, ion-ion interactions are thus limited to only a handful of nearest-neighbor pairs. We further update the results of the GW algorithm according to a "local electro-neutrality" condition, until convergence,

$$
z_{i}=-\operatorname{sign}\left(\sum_{j \neq i} \frac{z_{j} l_{B}}{\left|\mathbf{r}_{i}-\mathbf{r}_{j}\right|}\right) .
$$

Finally, the algorithm is accelerated by selecting the bisecting plane perpendicular to the first principal component of $S$.

\section{RESULTS}

Let us now apply the modified GW algorithm to test our main hypothesis, i.e., the charge distribution is determined by the ground state of a spin-glass Hamiltonian, given the positional configuration. A useful starting point is to examine systems in complete disorder by simulating hard-sphere liquids with different packing fractions (see details in the Supplemental Material [69]). Figure 2(b) shows the charge distribution around a central ion in the ground state. We notice an interesting trade-off between the distance of closest approach and overscreening. When ions are free to approach each other, it is almost always favorable for the nearest neighbor to be of opposite charge, regardless of other ions in the environment. Neighbors further away are much less correlated. As ionic radii increase, ions tend to be more evenly spaced and screening is shared by several neighbors; a longerrange oscillatory structure emerges.

\section{A. Ionic liquids}

Ionic liquids display a much longer correlation length. Data from scattering experiments as well as MD simulations

\section{Spin glass reconstruction of ionic liquids}
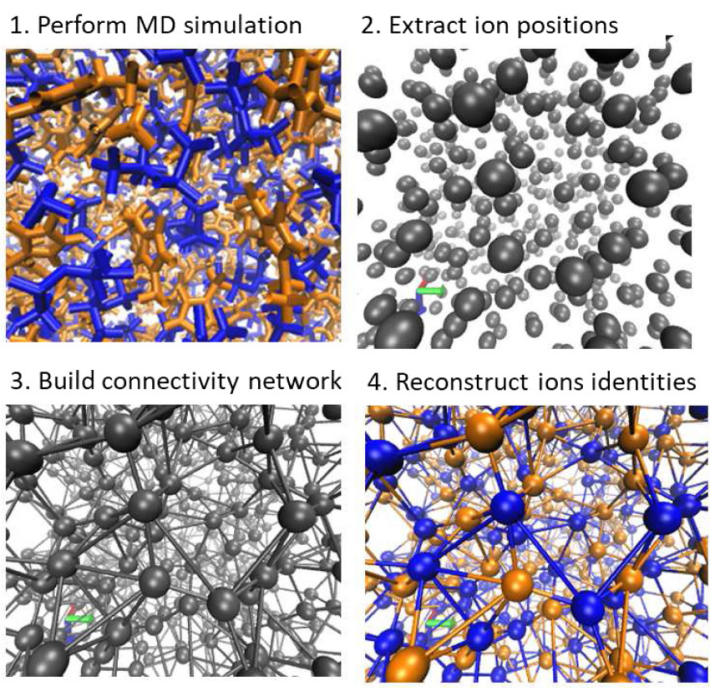

FIG. 1. Illustration of the reconstruction procedure. (1) The input is a full MD simulation of the ionic liquids. (2) The first step is to take a single snapshot, calculate the position of each molecule (as an average over its atomic positions), and delete the molecule identity. (3) Based on molecular positions, we construct a connectivity network by connecting each molecule to its nearest neighbors. (4) Minimizing the spin-glass Hamiltonian for the network yields identities for the molecules, marked by orange and blue in the figure. The minimization is carried for each snapshot separately. 
reveal complicated nanostructures $[17,29,50,52]$ with oscillations that span many neighbors. We simulate an EMIMTFSI ionic liquid to study these structures (see Supplemental Material for simulation details [69]). As illustrated in Fig. 1, the Hamiltonian is constructed from ionic positions extracted from MD simulation snapshots. The minimization scheme is carried separately for each snapshot and the results shown are averaged over all snapshots. Despite the complexity of the full atomistic MD simulations, the spin-glass model actually captures all the necessary physics: ionic valency almost exactly minimizes the Coulomb interactions. No other nonelectrostatic ingredient is needed to recover the charge density long-range correlations.

Figures 2(c) and 2(d) compares results from MD simulations to the spin-glass reconstruction process. In bulk simulations, we recover the exact charge of almost $98 \%$ of the ions. Consequently, the predicted charge distribution is almost indistinguishable from the simulated one [Fig. 2(c)]. This exceptional match hints towards a unique ground state and a high degree of order in the ionic positions. The reconstructed double-layer structure [Fig. 2(d)] fits reasonably well with the simulated EDL, despite completely neglecting the interaction with the electrode. For weak surface charges, this interaction is only a secondary effect, but will have to be considered as electrode charge increases. A weakly charge electrode also exhibits a dramatic overscreening. The first layer can have a charge that is, surprisingly, up to 15 times greater than the electrode charge. For comparison, BSK predicts an overscreening of only a few percent, which is more realistic for larger surface charges.

The spin-glass ground state aims to create long-range structures of alternating signs. Given the chance, a true longrange charge order would appear. Yet, this requires a high degree of order in the ionic positions. Even slight deviations from a perfect crystal structure lead to geometric frustrations: the pattern of alternating signs has to be broken in some direction [see Fig. 2(a) for illustration]. The positional disorder can be traced back to the particular size and shape of the ions $[63,64]$. Simple ions favor positional ordering and form ionic solids at room temperature, or long-range ordered liquids beyond the melting point, which are well captured by the spin-glass model (see below). This coupling of density and charge ordering has been extensively studied in the context of a solid-liquid phase transition [70-72]. Conversely, in complete disorder, correlations are limited to a few neighbors only [Fig. 2(b)]. In ionic liquids and especially near charged surfaces, partial disorder facilitates the large correlation lengths [Figs. 2(c) and 2(d)].

The order (or disorder) in ionic positions can take different forms: from short-range ion clusters to hydrogen-bond (H-bond) networks and micelle-like morphologies [73]. In some protic (H-bonding) ionic liquids, for example, complex nanostructures may be formed by the interplay of Coulomb forces and strong H-bond interactions [73]. However, this type

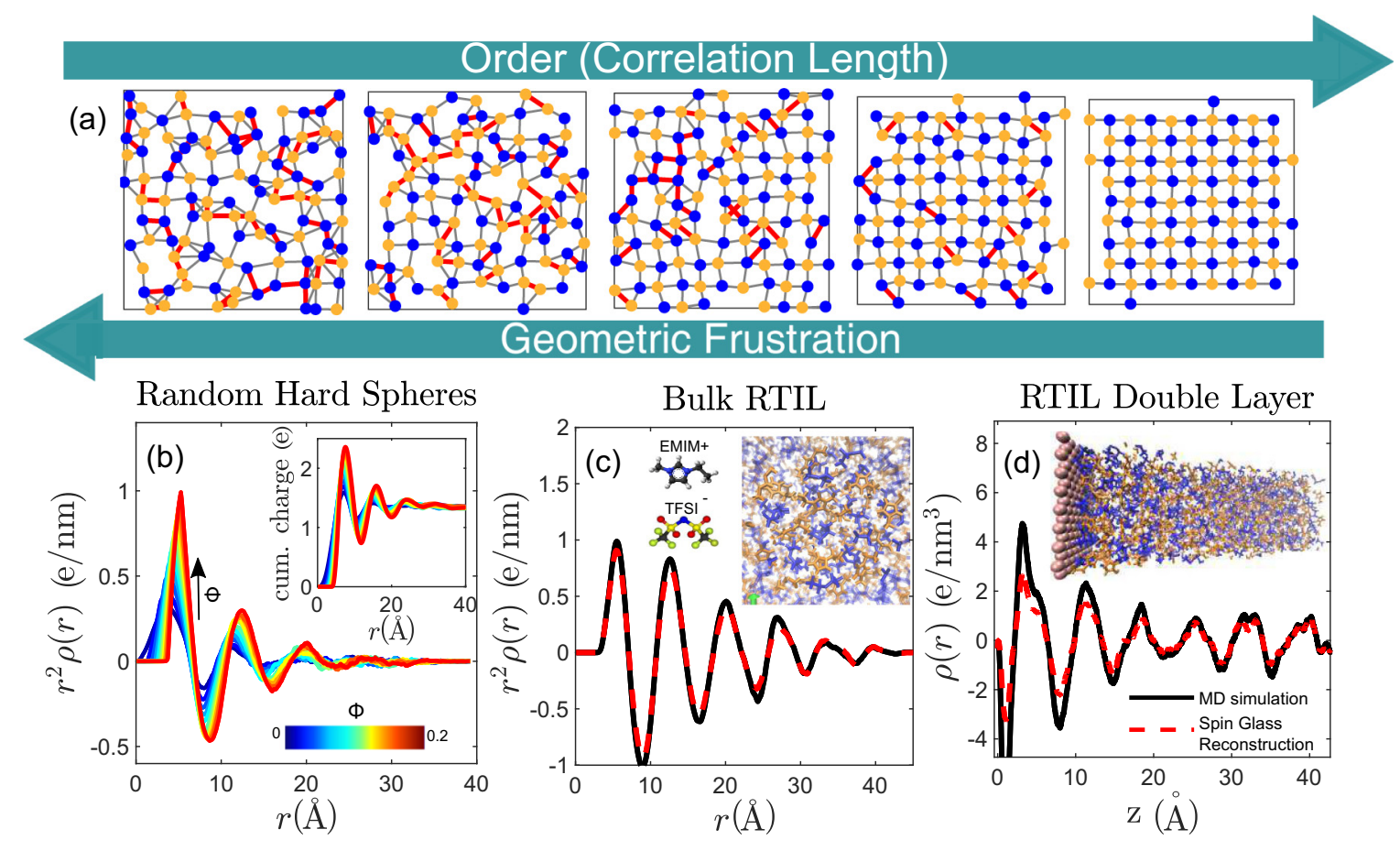

FIG. 2. Order vs frustration in ionic liquids. (a) Examples of a 2D Ising model, with different degrees of order. Red lines mark connections between parallel spins. The more ordered the system (right), the more connections are satisfied. (b)-(d) Examples of 3D spin glass with Coulomb interaction. (b) Charge distribution around a central ion in a random hard-sphere model, for different packing fractions $(\Phi)$, ranging from 0 to 0.2 in steps of 0.02 . Inset: cumulative charge distribution. Overscreening is defined as the maximum of this curve. (c) Charge distribution around a central TFSI ion in EMIM-TFSI, based on MD simulation (black line) and the spin-glass reconstruction (dashed red line). Inset: a snapshot from the MD simulation. (d) EMIM-TFSI charge density near a weakly charged surface $\left(0.01 \mathrm{C} / \mathrm{m}^{2}\right)$, based on $\mathrm{MD}$ simulation (black line) and spin-glass reconstruction (dashed red line) Inset: a snapshot from the MD simulation. 


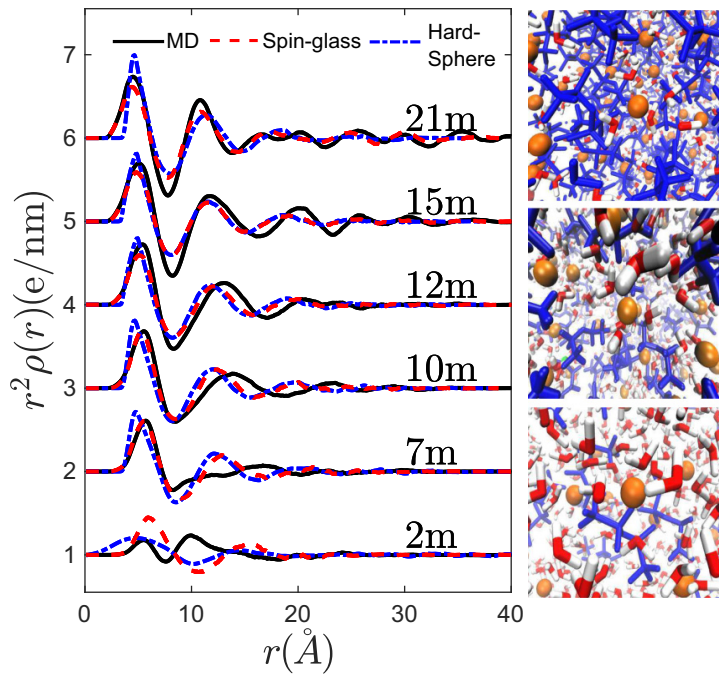

FIG. 3. LiTFSI in water: from concentrated electrolyte to WiSE. Charge distribution around a central TFSI ion is shown for different molality $(1 \mathrm{~mol} / \mathrm{kg}$ solvent $=1 \mathrm{~m})$, from 2 to $21 \mathrm{~m}$. Each graph is plotted with an offset of $1 e / \mathrm{nm}$. Results from MD simulation (solid black line) are compared with random hard-sphere toy model (dash-dotted blue line) and the spin-glass reconstruction (dashed red line). Snapshots are shown from the LiTFSI MD simulation for $2 \mathrm{~m}$ (bottom), $7 \mathrm{~m}$ (middle), and $21 \mathrm{~m}$ (top). The hard-sphere diameter equals $4.12 \AA$.

of behavior is not just unique to protic RTILs. For example, aprotic $\mathrm{C}_{n} \operatorname{mim}\left[\mathrm{PF}_{6}\right]$ ionic liquids tend to break into polar and nonpolar nanoregions [74]. In both cases, nonelectrostatic forces play an important role in determining the type of bulk nanostructures. We cannot rule out that these mechanisms affect charge ordering as well, but to demonstrate the generality of our method, we performed an additional simulation of trimethylammonium-triflate or TMA-OTF. TMA-OTF is an example of a protic ionic liquid, where $\mathrm{H}$ bonds play a major role. Protic and aprotic ionic liquids are the two main classifications of ionic liquids, and EMIM-TFSI is an example of the latter. The reconstruction results are shown in Fig. 4: despite the formation of $\mathrm{H}$ bonds, once the positions are known the charge ordering exactly followed the spin-glass minimization.

\section{B. Water-in-salt electrolyte}

Let us now examine MD simulations of the LiTFSI "waterin-salt electrolyte" (WiSE) at varying concentrations of salt. WiSEs have emerged as promising candidates to replace organic electrolytes in lithium-ion batteries [59-61]. They exhibit much shorter correlation lengths, even when the solvent concentration is small (Fig. 3). For moderate to high salt concentrations $(>5 \mathrm{~mol} / \mathrm{Kg})$, where ionic spacing is small compared to the Bjerrum length, our spin-glass framework is applicable.

Due in large part to ion size asymmetry, the spin-glass reconstruction only semiquantitatively matches the simulations. The high molality limit $(21 \mathrm{~m})$ is best reproduced by the minimization process, with about $80 \%$ of ionic charges recovered. Similarly to RTIL, the hidden positional order stands behind this unique and easily accessible ground state. With increasing water content $(21 \mathrm{~m} \rightarrow 7 \mathrm{~m})$, the order gradually disappears and we are only able to capture the general structure of the screening cloud. Upon decreasing ionic concentration further ( $7 \mathrm{~m}$ and especially $2 \mathrm{~m}$ ), thermal fluctuations become predominant and the spin-glass model breaks down. Yet, traditional mean-field models are unsuitable for that regime as well, and ion-specific effects determine the correlation function.

When ionic positions are disordered, the charge distribution matches the random hard-sphere model (dashed blue lines in Fig. 3). Similarities are even more pronounced when only considering ordering relative to neighbor number [69]. The reason for this high degree of disorder, compared with the RTIL, is twofold. First, there is a large positional entropy associated with small lithium ions, which is costly to suppress. Second, the solvent molecules weaken the electrostatic interactions. Maintaining a positional order is therefore unfavorable and the WiSE resembles a hard-sphere liquid.

\section{Molten salts}

The origin of disorder in high-temperature molten salts is the increased temperature. Due to their structure, simple salts form ionic solids at room temperature and keep a high degree of order even after their melting point. Molten salts have been extensively studied by restrictive primitive models (charged hard spheres) and their charge distribution is well described by integral equations methods [70]. We performed an $\mathrm{MD}$ simulation for a $\mathrm{NaCl}$-like molten salt at a temperature of $1500 \mathrm{~K}$. The simulation was performed using the same protocol as for the EMIM-TFSI, TMA-OTF, and LiTFSI electrolytes (see Supplemental Material for simulation details [69]). Our reconstruction scheme exactly reproduced the charge distribution around a central ion (Fig. 4). This excellent match is even better than the one found for ionic liquids or

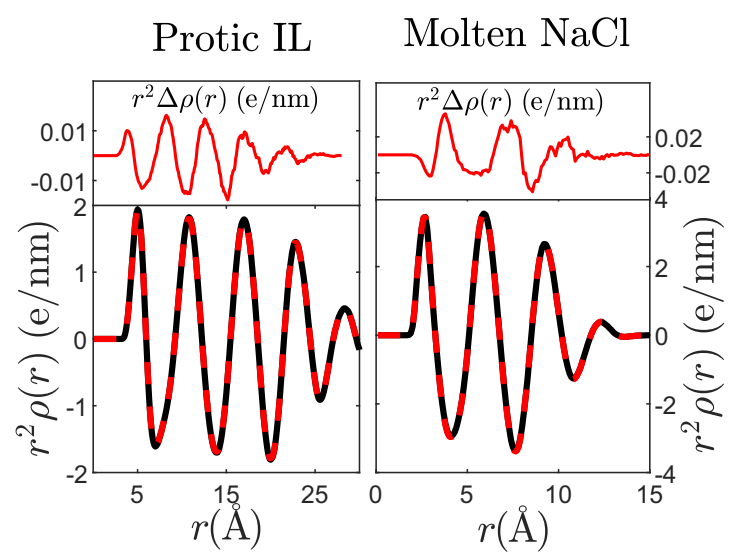

FIG. 4. Charge distribution around a central ion in a NaCl-type molten salt and TMA-OTF. Right: comparison of spin-glass reconstruction (dashed red line) vs MD simulation results (solid black line) for a molten salt. Left: testing the reconstruction scheme on a protic ionic liquid, TMA-OTF (dashed red line) with MD simulation results (solid black line). The difference in both cases is less than $1 \%$, as shown in the top figures. The small difference is due to the fact that an overwhelming majority of ions was identified correctly. 

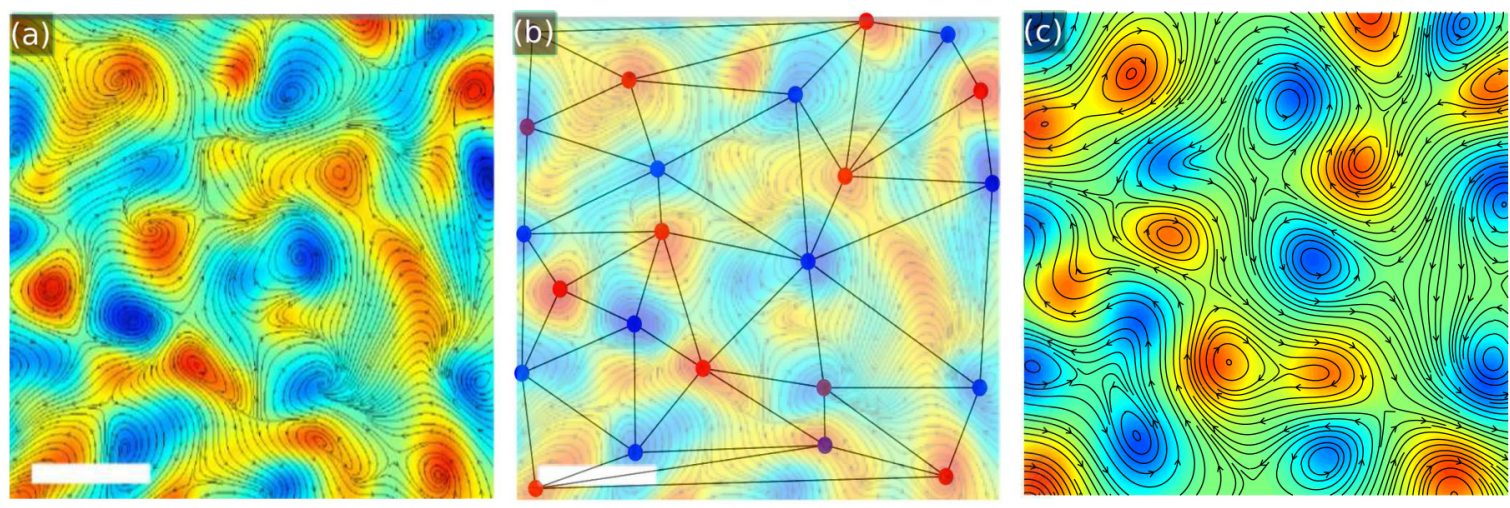

FIG. 5. Applying spin-glass reconstruction for swimming bacteria. (a) Snapshot of simulated flow field (adapted from [62] with permission). (b) A vortex network constructed by extracting the vortices' centers as nodes. The sign of each node, clockwise (red) or counterclockwise (blue) rotation, was derived from minimizing the spin-glass Hamiltonian and matches 19 out of the 23 nodes of the simulation. (c) Illustration of the reconstructed flow field, based on a superposition of independent Lamb-Oseen vortices.

water-in-salts and exemplifies how positional ordering of the molten salts generates an easily accessible ground state for the corresponding spin-glass Hamiltonian.

The success of our reconstruction scheme in capturing charge ordering for the variety of ionic systems displayed above demonstrates the potential of our method to apply to other ionic liquid systems, as well.

\section{Turbulence in bacterial suspensions}

Our analysis is not restricted to ionic liquids or a Coulombic system. Disordered systems with strong antiferromagnetic interactions are expected to show similar behavior. We demonstrate the generality of the spin-glass reconstruction scheme by considering turbulence in bacterial suspensions. A bacterial colony of Bacillus subtilis self-organizes into collective movement and forms vortices under confinement [62]. To minimize drag forces and reduce friction, adjacent vortices prefer to rotate in opposite directions. The details of this interaction follow complicated hydrodynamic equations, but as long as the antiferromagnetic interaction is strong, "spin" ordering is expected to dominate the emerging structure. We study the system with an effective spin-glass Hamiltonian, where the vortex directionality plays the role of spin and the positions of the vortices' cores are extracted from simulations. We use simulation data of swimming bacteria, adapted from [62]. Here, 23 positions of the vortices' core were extracted manually from a snapshot image of the simulated flow field [Fig. 5(a)].

We arbitrarily choose the same form of local interactions as the effective RTIL Hamiltonian [Eq. (4)], but restrict connectivity only to vortices that are in physical contact via Delaunay triangulation. The minimization process was carried out using the modified GW algorithm, omitting the last stage of requiring electroneutrality. Out of the 23 vortices, the directionality of 19 of them was reconstructed correctly [Fig. 5(b)]. To illustrate the reconstructed vorticity [Fig. 5(c)], we superimpose a Lamb-Oseen (Gaussian) vortex at each core location, with angular velocity $\Omega(r) \propto\left\{1-\exp \left[-\left(r / r_{m}\right)^{2}\right]\right\} / r^{2}$ and a radius of $r_{m}=25 \mu \mathrm{m}$. The nice qualitative match illustrates the universality of our approach. The emerging structures in disordered antiferromagnetic systems are governed by the geometry and are insensitive to details of their physical origin.

\section{DISCUSSION}

The spin-glass model is a strong-coupling theory. It simplifies the complex interactions in ionic liquids and water-in-salt electrolytes to a minimization of a Hamiltonian with only local interactions (though corrections for electroneutrality are required).

The correlation length is governed by geometric frustrations and increases with positional order. Such structures would emerge in any binary liquid with strong "antiferromagnetic" interactions and are not limited to Coulomb forces. Other examples include 2D vortex patterns that arise in superfluids or bacterial turbulence $[75,76]$. This is markedly different from the typical Debye-Huckel behavior, where electrostatic attraction competes with entropic "repulsion."

Interestingly, the positional order may not be apparent at first sight and does not significantly affect the densitydensity pair-correlation function. Nevertheless, as we show via the minimization process, long-range charge ordering is a manifestation of the hidden positional ordering. The ionic positions, as disordered as they may seem, carry all the information about the ionic identities. It is not surprising that charge and density ordering are coupled: ionic positions are determined by the charges, and vice versa. However, our analysis offers a different way to understand the charge distribution in the bulk and reveals its intimate link to the positional configurations.

For solvent-free ionic liquids, the ground state of the spinglass Hamiltonian is easily accessible and correlations are long range. This might be the onset of a true long-range order in ionic crystals. Room-temperature ionic crystals have much stronger interactions due to their small size, but we speculate that a similar regime of hidden positional order must exist and play a role in the thermodynamics of melting. As solvent content increases, the energy landscape becomes more rugged, yet the system is still described well by its ground state and nonidealities are safely neglected. Eventually, in the moderately concentrated electrolyte regime $(<7 \mathrm{~m})$, 
thermal fluctuations as well as ion- and solvent-specific effects are dominating, and the spin-glass approach is no longer valid.

While further exploration is needed to determine the generality of our approach, we have shown it to work well for four classes of Coulombic liquids: priotic ILs, a-priotic ILs, molten salts, and water-in-salt electrolytes. In each case, the positional ordering was different, but the charge ordering was shown to be uniquely determined via spin-glass minimization. We note, however, that we only focus on the nanometer length scale, which is suitable for atomistic simulations. Longerrange phenomena, such as extremely long-range charge correlations, are beyond the scope of this work. Interestingly, we were not able to observe underscreening [43], despite its emergence in the relevant length scales.

Another important limitation of our model is that it is inherently symmetric in size and shape. In many cases, it is the asymmetry of the ions that favors disordered structures and makes them good ionic liquids in the first place. Remarkably, even in extremely asymmetric cases such as LiTFSI, the spin-glass Hamiltonian qualitatively correctly captures the charge distribution. This is in part due to a universal behavior of complete disorder, which does not depend on the details of the materials.

Though we do not offer a general theory of ionic liquids and concentrated electrolytes, we believe our observations highlight the important physics. The close interplay between the positional configurations and charge ordering, which plays an important role in long-range ordering near phase transitions, is observed to be significant even in partial or complete disorder. Geometric frustrations and positional ordering determine the correlations lengths and might be considered as some of the underlying microscopic driving forces to include for accurate continuum models.

\section{ACKNOWLEDGMENT}

This work was supported by the Amar G. Bose Research Grant.
[1] M. V. Fedorov and A. A. Kornyshev, Chem. Rev. 114, 2978 (2014).

[2] G.-T. Kim, S. S. Jeong, M.-Z. Xue, A. Balducci, M. Winter, S. Passerini, F. Alessandrini, and G. B. Appetecchi, J. Power Sources 199, 239 (2012).

[3] B. Scrosati and J. Garche, J. Power Sources 195, 2419 (2010).

[4] B. Garcia, S. Lavallée, G. Perron, C. Michot, and M. Armand, Electrochim. Acta 49, 4583 (2004).

[5] P. Simon and Y. Gogotsi, Nat. Mater. 7, 845 (2008).

[6] P. Debye and E. Hückel, Phys. Z. 24, 185 (1923).

[7] M. Z. Bazant, M. Sabri, B. D. Storey, and A. Ajdari, Adv. Colloid Interface Sci. 152, 48 (2009).

[8] I. Borukhov, D. Andelman, and H. Orland, Phys. Rev. Lett. 79, 435 (1997).

[9] J. J. Bikerman, Philos. Mag. 33, 384 (1942).

[10] M. S. Kilic, M. Z. Bazant, and A. Ajdari, Phys. Rev. E 75, 021502 (2007).

[11] A. A. Kornyshev, J. Phys. Chem. B 111, 5545 (2007).

[12] B. V. Derjaguin and L. D. Landau, Acta Phys. Chim. USSR 14, 633 (1941)

[13] E. J. W. Verwey and J. T. G. Overbeek, Theory of the Stability of Lyophobic Colloids (Elsevier, New York, 1948).

[14] Z. A. Goodwin, G. Feng, and A. A. Kornyshev, Electrochim. Acta 225, 190 (2017).

[15] Z. A. H. Goodwin and A. A. Kornyshev, Electrochem. Commun. 82, 129 (2017).

[16] R. M. Adar, T. Markovich, and D. Andelman, J. Chem. Phys. 146, 194904 (2017).

[17] N. Gavish, D. Elad, and A. Yochelis, J. Phys. Chem. Lett. 9, 36 (2018).

[18] A. Abrashkin, D. Andelman, and H. Orland, Phys. Rev. Lett. 99, 077801 (2007).

[19] E. Gongadze, U. van Rienen, V. Kralj-Iglič, and A. Iglič, Comput. Methods Biomech. Biomed. Eng. 16, 463 (2013).

[20] R. R. Netz and H. Orland, Europhys. J. E 1, 203 (2000).

[21] M. A. Gebbie, M. Valtiner, X. Banquy, E. T. Fox, W. A. Henderson, and J. N. Israelachvili, Proc. Natl. Acad. Sci. USA 110, 9674 (2013).
[22] A. A. Lee, D. Vella, S. Perkin, and A. Goriely, J. Phys. Chem. Lett. 6, 159 (2015).

[23] M. V. Fedorov, N. Georgi, and A. A. Kornyshev, Electrochem. Commun. 12, 296 (2010).

[24] M. V. Fedorov and A. A. Kornyshev, Electrochim. Acta 53, 6835 (2008).

[25] C. D. Santangelo, Phys. Rev. E 73, 041512 (2006).

[26] M. M. Hatlo and L. Lue, Europhys. Lett. 89, 25002 (2010).

[27] M. Z. Bazant, B. D. Storey, and A. A. Kornyshev, Phys. Rev. Lett. 106, 046102 (2011).

[28] J.-L. Liu and B. Eisenberg, J. Phys. Chem. B 117, 12051 (2013).

[29] N. Gavish and A. Yochelis, J. Phys. Chem. Lett. 7, 1121 (2016).

[30] R. Blossey, A. C. Maggs, and R. Podgornik, Phys. Rev. E 95, 060602(R) (2017).

[31] R. Downing, B. K. Berntson, G. V. Bossa, and S. May, J. Chem. Phys. 149, 204703 (2018).

[32] A. A. Kornyshev, A. I. Rubinshtein, and M. A. Vorotyntsev, J. Phys. C: Solid State Phys. 11, 3307 (1978).

[33] A. Hildebrandt, R. Blossey, S. Rjasanow, O. Kohlbacher, and H.-P. Lenhof, Phys. Rev. Lett. 93, 108104 (2004).

[34] D. W. Lee, D. J. Im, and I. S. Kang, Langmuir 29, 1875 (2013).

[35] A. Yochelis, J. Phys. Chem. C 118, 5716 (2014).

[36] G. J. Moon, M. M. Ahn, and I. S. Kang, Phys. Rev. E 92, 063020 (2015).

[37] J.-L. Liu and B. Eisenberg, Chem. Phys. Lett. 637, 1 (2015).

[38] A. A. Lee, S. Kondrat, D. Vella, and A. Goriely, Phys. Rev. Lett. 115, 106101 (2015).

[39] P. H. R. Alijó, F. W. Tavares, E. C. Biscaia Jr., and A. R. Secchi, Electrochim. Acta 152, 84 (2015).

[40] X. Jiang, Y. Liu, and R. Qiao, J. Phys. Chem. C 120, 4629 (2016).

[41] X. Jiang, J. Huang, H. Zhao, B. G. Sumpter, and R. Qiao, J. Phys.: Condens. Matter 26, 284109 (2014).

[42] R. F. Stout and A. S. Khair, J. Fluid Mech. 752, R1 (2014).

[43] C. S. Perez-Martinez, A. M. Smith, and S. Perkin, Discuss. Faraday Soc. 199, 239 (2017).

[44] A. M. Smith, A. A. Lee, and S. Perkin, Phys. Rev. Lett. 118, 096002 (2017). 
[45] A. A. Lee, C. S. Perez-Martinez, A. M. Smith, and S. Perkin, Phys. Rev. Lett. 119, 026002 (2017).

[46] V. Ivaništšev, S. O'Connor, and M. V. Fedorov, Electrochem. Commun. 48, 61 (2014).

[47] V. Ivaništšev, K. Kirchner, T. Kirchner, and M. V. Fedorov, J. Phys.: Condens. Matter 27, 102101 (2015).

[48] B. Rotenberg and M. Salanne, J. Phys. Chem. Lett. 6, 4978 (2015).

[49] A. A. Kornyshev and R. Qiao, J. Phys. Chem. C 118, 18285 (2014).

[50] A. Triolo, O. Russina, H.-J. Bleif, and E. Di Cola, J. Phys. Chem. B 111, 4641 (2007).

[51] J. N. Canongia Lopes, M. F. Costa Gomes, and A. A. H. Pádua, J. Phys. Chem. B 110, 16816 (2006).

[52] R. Atkin and G. G. Warr, J. Phys. Chem. B 112, 4164 (2008).

[53] S. F. Edwards and P. W. Anderson, J. Phys. F: Met. Phys. 5, 965 (1975).

[54] M. X. Goemans and D. P. Williamson, J. ACM 42, 1115 (1995).

[55] R. Palm, H. Kurig, K. Tõnurist, A. Jänes, and E. Lust, Electrochem. Commun. 22, 203 (2012).

[56] C. Largeot, C. Portet, J. Chmiola, P.-L. Taberna, Y. Gogotsi, and P. Simon, J. Am. Chem. Soc. 130, 2730 (2008).

[57] H. Matsumoto, H. Sakaebe, and K. Tatsumi, J. Power Sources 146, 45 (2005).

[58] S. Seki, Y. Kobayashi, H. Miyashiro, Y. Ohno, A. Usami, Y. Mita, N. Kihira, M. Watanabe, and N. Terada, J. Phys. Chem. B 110, 10228 (2006).

[59] L. Suo, Y.-s. Hu, H. Li, M. Armand, and L. Chen, Nat. Commun. 4, 1 (2013).

[60] L. Suo, O. Borodin, T. Gao, M. Olguin, J. Ho, X. Fan, C. Luo, C. Wang, and K. Xu, Science 350, 938 (2015).
[61] M. McEldrew, Z. A. H. Goodwin, A. A. Kornyshev, and M. Z. Bazant, J. Phys. Chem. Lett. 9, 5840 (2018).

[62] J. Dunkel, S. Heidenreich, K. Drescher, H. H. Wensink, M. Bär, and R. E. Goldstein, Phys. Rev. Lett. 110, 228102 (2013).

[63] A. Perera and R. Mazighi, J. Mol. Liq. 210, 243 (2015).

[64] A. Perera, Phys. Chem. Chem. Phys. 19, 1062 (2017).

[65] M. R. Garey and D. S. Johnson, Computers and Intractability: A Guide to the Theory of NP-completeness, Vol. 29 (Freeman, San Francisco, 1979).

[66] S. Chandra, Phys. Rev. E 77, 021125 (2008).

[67] M. C. Grant and S. P. Boyd, Recent Advances in Learning and Control (Springer, London, 2008).

[68] M. Grant and S. Boyd, CVx: Matlab software for disciplined convex programming, version 2.0 beta, http://cvxr.com/cvx (unpublished).

[69] See Supplemental Material at http://link.aps.org/supplemental/ 10.1103/PhysRevMaterials.3.055606 for a detailed description of the simulations and for a detailed analysis of the screening cloud in WiSE.

[70] J. P. Hansen and I. R. McDonald, Phys. Rev. A 11, 2111 (1975).

[71] J.-L. Barrat, J. Phys. C: Solid State Phys. 20, 1031 (1987).

[72] O. Patsahan and A. Ciach, J. Phys.: Condens. Matter 19, 236203 (2007).

[73] R. Hayes, G. G. Warr, and R. Atkin, Chem. Rev. 115, 6357 (2015).

[74] J. N. Canongia Lopes and A. A. Pádua, J. Phys. Chem. B 110, 3330 (2006).

[75] J. R. Abo-Shaeer, C. Raman, J. M. Vogels, and W. Ketterle, Science 292, 476 (2001).

[76] H. Wioland, F. G. Woodhouse, J. Dunkel, and R. E. Goldstein, Nat. Phys. 12, 341 (2016). 